\title{
Simulation of Incompressible Non-Newtonian Flows Through Channels with Sudden Expansion Using the Power-Law Model ${ }^{1}$
}

R. MANICA 2 , A.L. de BORTOLI ${ }^{3}$, Progama de Pós-Graduação em Matemática Aplicada, IM-DMPA, UFRGS, Av. Bento Gonçalves 9500, 91509-900 Porto Alegre, RS, Brazil.

\begin{abstract}
The goal of this work is to analyze incompressible Newtonian and nonNewtonian flows through channels with sudden expansion. The governing equations are solved using the finite-differences explicit Runge-Kutta time-stepping scheme in nondimensionalized form in which continuity and momentum are solved simultaneously along the grid points. The power-law model is applied to predict pseudoplastic (shear-thinning) and dilatant (shear-thickening) behavior in such expansions. The critical Reynolds number, in which the solution becomes asymmetric, is analyzed. Numerical results for a 3:1 expansion show good agreement with other numerical tests found in the literature for Reynolds numbers ranging from 40 to 140 for Newtonian flow. For the non-Newtonian case, a comparison with an analytical solution is presented.
\end{abstract}

\section{Introduction}

In the recent years, it has been of great interest to study systems experiencing transition; the flow in a symmetric sudden expansion is an example. It has been observed experimentally that, below a certain critical Reynolds number, the flow is symmetric. This symmetric flow becomes unstable once the Reynolds number exceeds the critical value, and a pair of steady asymmetric vortices is observed as one recirculation region grows at the expense of the other; a symmetric-breaking bifurcation takes place. As the Reynolds number increases further, the flow may become three-dimensional and oscillatory [2].

Expansions have important applications in engineering processes like in refrigeration, extrusion and free jets. The study of Newtonian fluid flows through a sudden expansion of various ratios and conditions is a classical problem which has been analyzed by many workers. Much information about this flow are known, like the critical Reynolds and bifurcation phenomena. For non-Newtonian flows, such investigation is recent and there isn't much information about it. A better understanding

\footnotetext{
${ }^{1}$ Trabalho apresentado no CNMAC.

2 tcheli@mat.ufrgs.br; Bolsista do CNPq

${ }^{3}$ dbortoli@mat.ufrgs.br
} 
of non-Newtonian flow through sudden expansions should lead to both the design and development of hydrodynamically more efficient processes and to an improved quality control of the final products.

Non-Newtonian fluids are usually considered to be those when the relation connecting shear stress and shear rate is not linear; that is, the 'viscosity' of a nonNewtonian fluid is not constant at a given temperature and pressure, but depends on the rate of shear or on the previous kinematic history of the fluid [7]. In this way, there isn't a constitutive relation able to predict all non-Newtonian behavior that can occur. So, a lot of models were developed for predicting non-Newtonian effects like the Maxwell, the generalized Newtonian liquid (GNL) [8] [1] and the models based on differential and integral constitutive equations. The power-law model [4], which is a special case of the GNL, is applied to predict pseudoplastic and dilatant behavior.

The typical curve relating stress-deformation rate for pseudoplastic fluids indicates that the viscosity falls progressively with shear rate and the flow curve becomes linear only at very high shear rates. This behavior is characteristic of high polymers, polymer solutions and many suspensions. For dilatant fluids, the viscosity increases when the rate of shear is increased [3].

In the following, the governing equations, solution technique, and numerical results are presented.

\section{Governing Equations and Solution Technique}

The governing equations for a two-dimensional, laminar, incompressible flow in nondimensionalized form are written as follows

$$
\begin{gathered}
\frac{\partial u}{\partial x}+\frac{\partial v}{\partial y}=0, \\
\frac{\partial u}{\partial t}+u \frac{\partial u}{\partial x}+v \frac{\partial u}{\partial y}=-\frac{\partial p}{\partial x}+\frac{1}{R e}\left\{\frac{\partial \tau_{x x}}{\partial x}+\frac{\partial \tau_{x y}}{\partial y}\right\}, \\
\frac{\partial v}{\partial t}+u \frac{\partial v}{\partial x}+v \frac{\partial v}{\partial y}=-\frac{\partial p}{\partial y}+\frac{1}{R e}\left\{\frac{\partial \tau_{x y}}{\partial x}+\frac{\partial \tau_{y y}}{\partial y}\right\},
\end{gathered}
$$

where $u$ and $v$ are the velocity vector components in $x$ and $y$ directions, $p$ the pressure (coming from a Poisson equation), $t$ the time, $\tau_{i j}$ the viscous part of stress tensor, and Re the generalized Reynolds number, which is given by

$$
R e=\frac{\rho V^{2-n} H^{n}}{K},
$$

with $\rho$ the fluid density, $V$ the characteristic velocity, $H$ the height of the inlet cross-section and $K$ the consistency index.

For the power-law model the 'extra' stress tensor is written as

$$
\tau=2 \mu_{e f} D,
$$


where $D$ is the rate of strain tensor given by $D=\left[\nabla u+(\nabla u)^{T}\right]$ and $\mu_{e f}$ the effective viscosity which comes from

$$
\mu_{e f}=\left(\frac{1}{2} D_{I I}\right)^{(n-1) / 2}
$$

where $n$ is the power-law index and $D_{I I}$ the second invariant of the strain rate given by

$$
\frac{1}{2} D_{I I}=2\left[\left(\frac{\partial v}{\partial y}\right)^{2}+\left(\frac{\partial u}{\partial x}\right)^{2}\right]+\left(\frac{\partial v}{\partial x}+\frac{\partial u}{\partial y}\right)^{2} .
$$

For solving this set of governing equations, methods such as finite elements, finite volume and finite differences are usually employed. All of them have their own advantages and disadvantages and are successfully used by known researchers all over the world. Here, the node-centered central finite differences scheme is employed for solving the governing equations, as shown in figure 1; such method is preferred because of its simplicity.

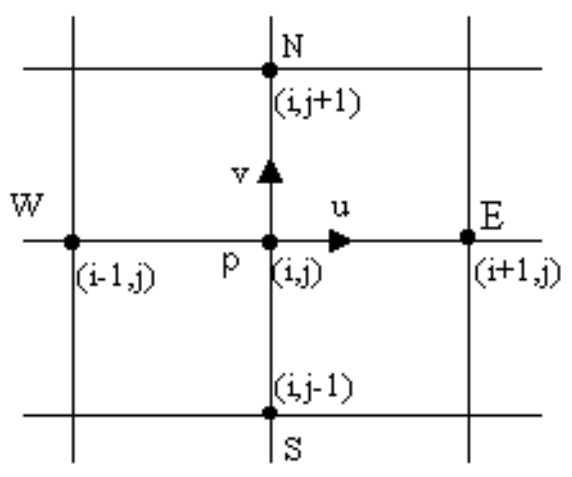

Figure 1: Node-centered arrangement

Convective and diffusive terms of these equations are in finite differences approximated according to, for example,

$$
\begin{gathered}
\frac{\partial u}{\partial x}=\frac{u_{i+1, j}-u_{i-1, j}}{2 \Delta x}+O\left(\Delta x^{2}\right), \\
\frac{\partial^{2} u}{\partial x^{2}}=\frac{u_{i+1, j}-2 u_{i, j}+u_{i-1, j}}{\Delta x^{2}}+O\left(\Delta x^{2}\right) .
\end{gathered}
$$

Now, the grid and boundary conditions are presented. Figure 2 shows an amplification of the structured grid of $112 \times 95$ points, for a $3: 1$ expansion, where the refinement at the expansion zone is shown. The domain is 30 dimensionless units long and 3 dimensionless units wide. A grid stretching at corners is recommended, 
because of the pressure and velocity gradients, which can be a serious drawback to the convergence of the numerical procedure. It is important to note that the boundary has corners in the expansion zone and some care need to be taken when the boundary conditions are applied.

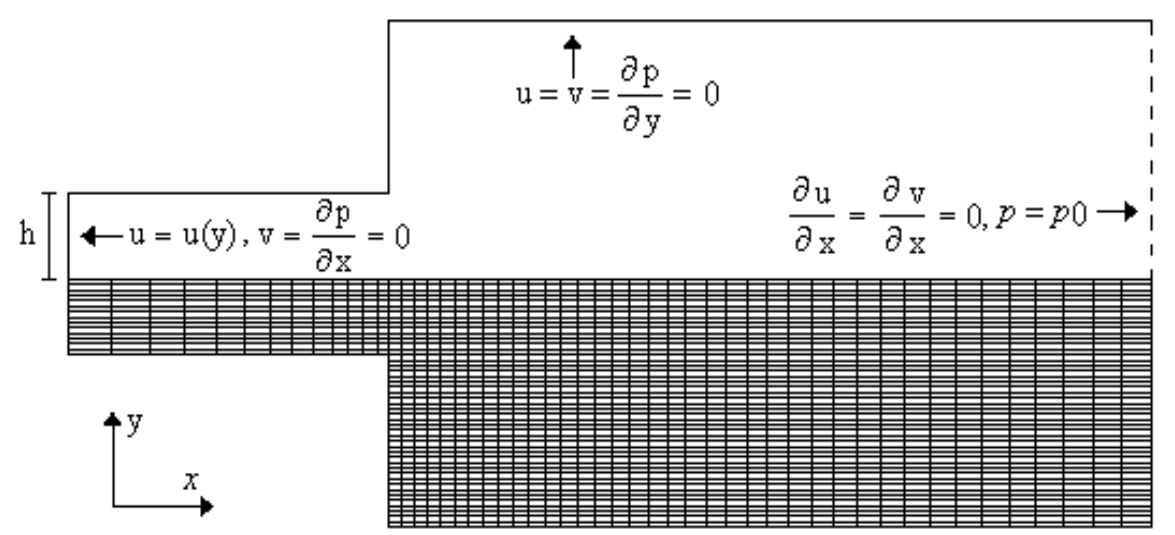

Figure 2: Grid amplification - 112x95 points and boundary conditions

The boundary conditions are defined as follows: at the inlet parabolic condition for velocity and extrapolation for pressure, at the walls no-slip conditions for velocity and extrapolation for pressure and at the output extrapolation for velocity and a fixed pressure $p 0$, which is its initial value. These conditions seem to be good for convergence.

The set of approximated governing equations is solved using the low-storage simplified Runge-Kutta time-stepping scheme to solve for velocities as follow

$$
\begin{gathered}
\vec{W}_{i j}^{0}=\vec{W}_{i j}^{n}, \\
\vec{W}_{i j}^{k}=\vec{W}_{i j}^{0}-\alpha_{k} \Delta t \vec{R}_{i j}^{k-1}, \\
\vec{W}_{i j}^{n+1}=\vec{W}_{i j}^{k},
\end{gathered}
$$

where $\vec{W}=(u, v)^{T}$ is the vector of convective variables, $\Delta t$ the time step, $\vec{R}_{i j}$ residual vector, and $\alpha_{k}$ the time coefficients, which for the 3 stage second order case are $\alpha_{1}=1 / 2, \alpha_{2}=1 / 2$, and $\alpha_{3}=1$. The simplified Runge-Kutta method is characterized by its low operation count and low storage requirements; more than two stages are employed to extend the stability region. For pressure the Relaxed Gauss-Seidel approach (SUR) is applied with $w=0.9$. 


\section{Numerical Results}

Tests are carried out for a $3: 1$ expansion for Reynolds numbers ranging from 40 to 140 . Figure 3 shows the steady solution for the Newtonian case. The results for $R e=40$ (3.a) and $R e=50$ (3.b) compare very well with those obtained by Luo [5]. The results for $R e=80$ (3.c) and $R e=140$ (3.d) compare with those obtained by Fearn et al. [2]; they found that for $R e>140$ the flow becomes unsteady. As can be seen, the recirculations for $R e=40$ are symmetric and for $R e=50$ an asymmetry appears. It is evident that there is a critical Reynolds $\left(R e_{c}\right)$ in which the solution becomes asymmetric.

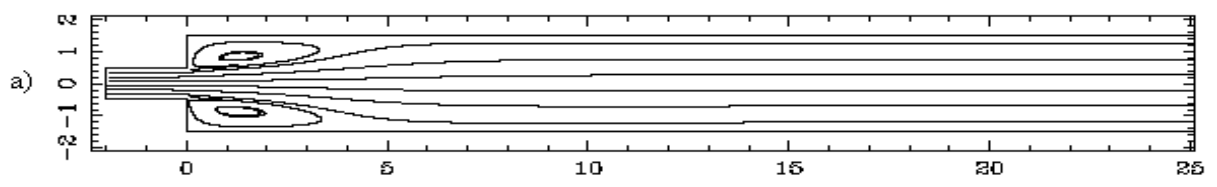

b)

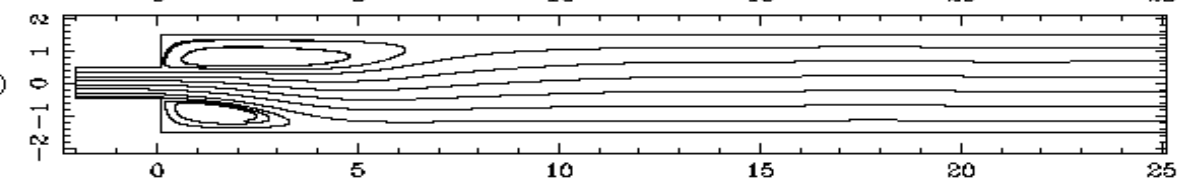

c)

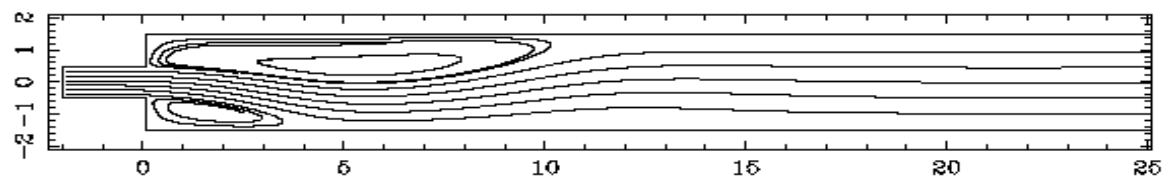

d)
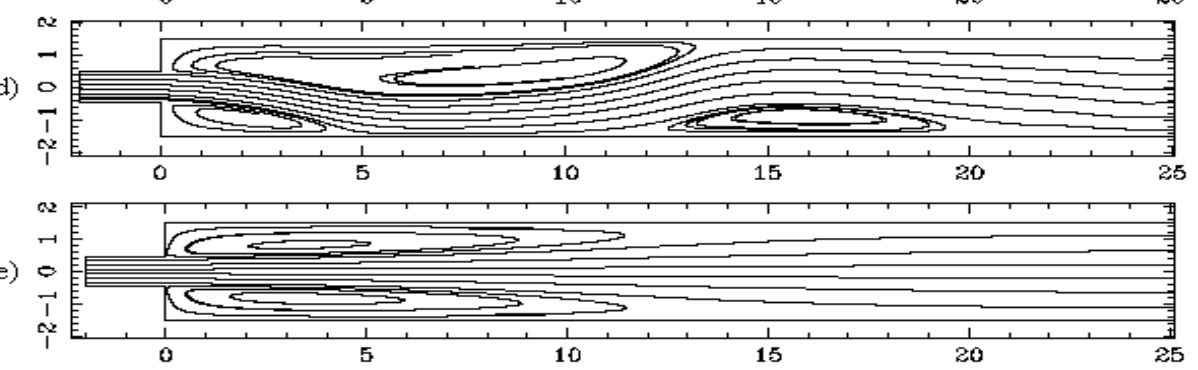

Figure 3: Streamlines for newtonian flow; a) $R e=40$, b) $R e=50$, c) $R e=80$, d) $R e=140$ and e) $R e=140$ (symmetric solution)

In the work of Fearn et al. [2], an expansion with the same conditions was analyzed. They made both numerical simulations and experiments and obtained $R e_{c}=40.45$ and 47.3, respectively. Luo [5] found $R e_{c}=46.19$. So, the results presented in this work are in agreement with those works.

According to Fearn et al. [2], above the $R e_{c}$ the equations have three solutions, of which one of them is unstable. As a consequence of the geometry symmetry, the two stable solutions above $R e_{c}$ are a pair of asymmetric solutions of opposite senses 
with respect to the line of symmetry. Figure (3.e) presents the symmetric solution for $R e=140$; when a perturbation occurs, the flow turns unstable.

Pak et al. [6] made experimental tests using a Newtonian fluid, a purely viscous non-Newtonian fluid (pseudoplastic) and a viscoelastic non-Newtonian fluid. They found out that for the purely viscous non-Newtonian fluid the reattachment length and the size of the vortex are almost the same that for a Newtonian fluid in the laminar situation. Otherwise, the flow becomes turbulent earlier with a decrease of the power-law exponent $n$. For the viscoelastic flow, the reattachment lengths for the laminar flow are shorter than those for Newtonian flow for the same Reynolds Number.

Figure 4 shows a comparison using $R e=40$ for a pseudoplastic with $n=0.5$, a Newtonian $(n=1)$, and a dilatant fluid with $n=2$. As the flow is symmetric, only the upper part is shown. The recirculations present differences in size: for the pseudoplastic case the vortex is rounder than for the Newtonian, and for the dilatant it is thinner. Nevertheless, the $R e_{c}$ for these cases does not have significant changes compared to the Newtonian case.
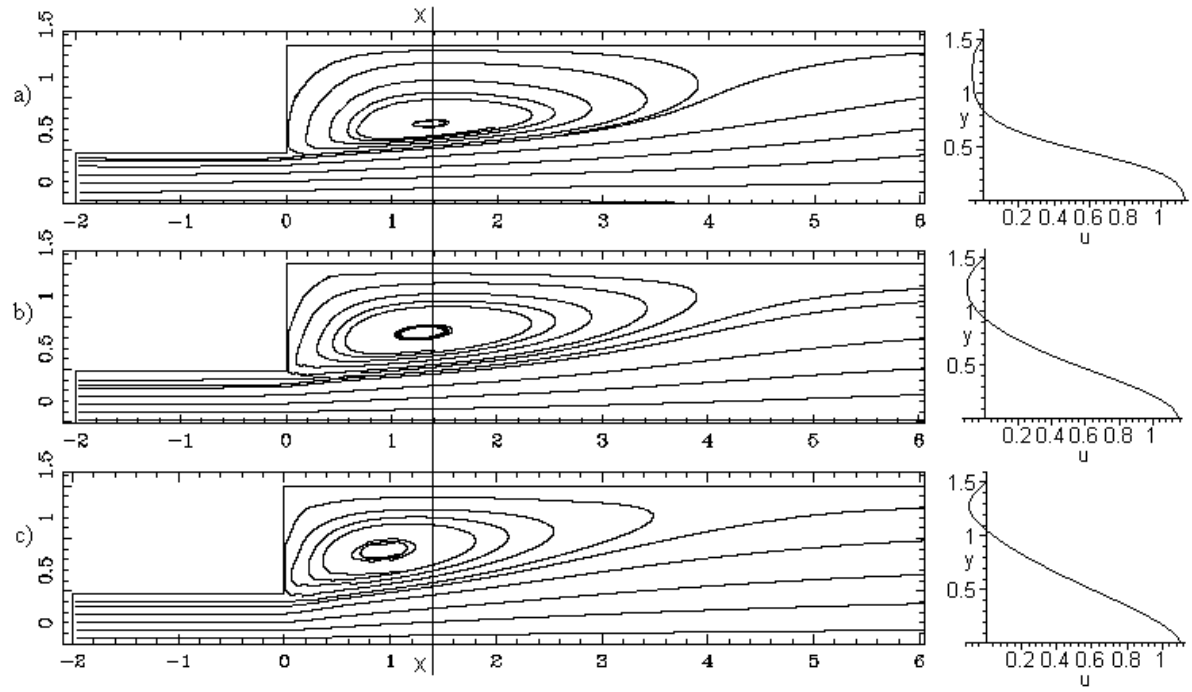

Figure 4: Streamlines and corresponding velocity profiles (at line $\mathrm{x}-\mathrm{x}$ ) comparison for $R e=40$; a) pseudoplastic $(n=0.5)$, b) Newtonian $(n=1)$ and c) dilatant $(n=2)$

Tanner [7] presented an adimensionalized analytical solution for the velocity profiles for a channel. The output profiles obtained for the solutions shown in figure 4 are compared with analytical profiles indicated in figure 5, and a good agreement is noticed. These results prove that the code can well represent power-law flows in 
channels. The analytical solution is given by

$$
u=\left(\frac{n}{n+1}\right)\left(\frac{p^{\prime}}{2 K}\right)^{1 / n} H^{1+1 / n}\left[1-\left(\frac{y}{H}\right)^{1+1 / n}\right],
$$

where $p^{\prime}$ is the magnitude of the pressure drop per unit length in the axial direction and $H$ the channel width. Dimensionless velocity profiles $u(y) / \bar{u}$ for $n=0.5,1$. and 1.5 are plotted, where the mean velocity is $\bar{u}=Q / \pi H^{2}$. The rate of discharge is given by

$$
Q=\frac{\pi n}{3 n+1}\left(\frac{p^{\prime}}{2 K}\right)^{1 / n} H^{3+1 / n}
$$

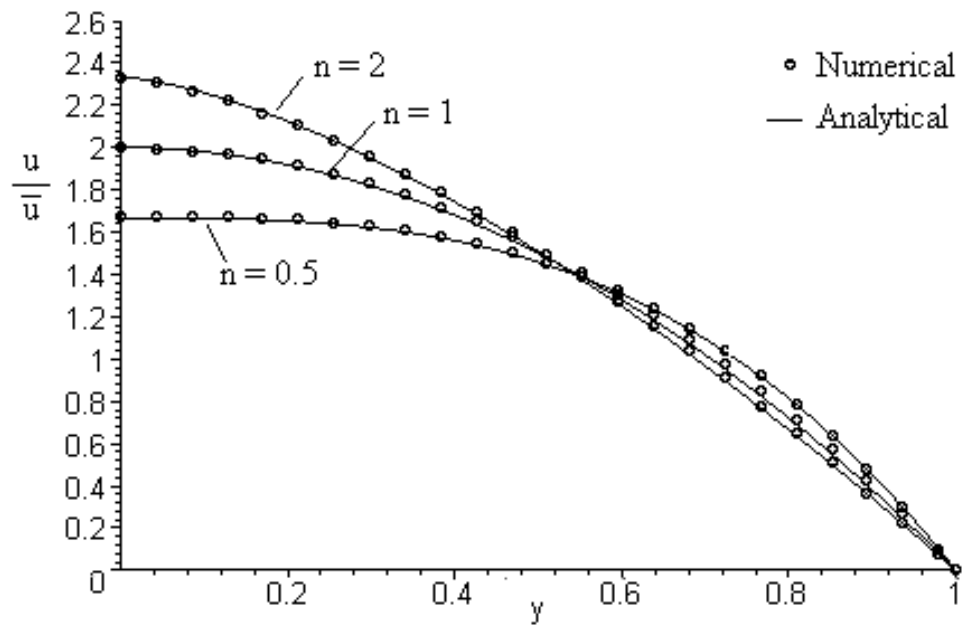

Figure 5: Comparison of numerical and analytical velocity profiles

\section{Conclusions}

Numerical results show that the method used here is able to well represent incompressible Newtonian as well as non-Newtonian (power-law) flows in expansions at low Reynolds numbers. When increasing the Reynolds number, there will appear more instabilities and the problem probably becomes tridimensional and time dependent (with oscillating behavior).

The power-law model is simple to be employed and represents well some nonNewtonian behavior. Otherwise, for more complex non-Newtonian flow the HerschelBulkley or Maxwell models can be employed; it is the goal of our future works. Besides, there is a notable lack of experimental data about this non-Newtonian 
problem.

It is the opinion of the authors that the obtained results are encouraging. However, much work still remains to compare such complex non-Newtonian flow behavior, where the investigations are still recent and there isn't much available information in the literature.

Resumo. O objetivo deste trabalho é analisar fluxos de fluidos newtonianos e não newtonianos em canais com expansão brusca. As equações governantes são resolvidas em diferenças finitas usando o esquema explícito de Runge-Kutta na forma adimensionalizada onde a continuidade e a quantidade de movimento são resolvidas simultaneamente ao logo dos pontos da malha. O modelo power-law é aplicado para prever comportamentos pseudoplásticos e dilatantes em tais expansões. O Reynolds crítico, em que o fluxo se torna assimétrico, é analisado. Resultados numéricos para uma razão de expansão de $3: 1$ apresentam boa concordância com dados numéricos encontrados na literatura para números de Reynolds variando entre 40 e 140 para fluxo newtoniano. Para o caso não newtoniano, uma comparação com a solução analítica é apresentada.

\section{References}

[1] A.N. Alexandrou, T.M. Mcgilvreay, G. Burgos, Steady Hershel-Bulkley Fluid Flow in Three-dimensional Expansions, Journal of Non-Newtonian Fluid Mechanics, 100 (2001), 77-96.

[2] R. Fearn, T. Mullin and K.A. Cliffe, Nonlinear Flow Phenomena in a Symmetric Sudden Expansion, Journal of Fluid Mechanics, 211 (1990), 595-608.

[3] J. Ferguson and Z. Kemblowki, "Applied Fluid Rheology", Elsevier Cambridge, 1991.

[4] L. Lefton and D. Wei, A Penalty Method for Approximations of the Stationary Power-Law Stokes Problem, Eletronic Journal of Differential Equations, $\mathbf{7}$ (2001), 1-12.

[5] L. Luo, Symmetric Breaking of Flow in 2D Symmetric Channels: Simulation by Lattice-Boltzmann Method, International Journal of Modern Physics, 8 (1997), 859-867.

[6] B. Pak, Y.I. Cho and S.U.S. Choi, Separation and Reattachment of NonNewtonian Fluid Flows in a Sudden Expansion Pipe, Journal of NonNewtonian Fluid Mechanics, 37 (1990), 175-199.

[7] R. I. Tanner, "Engineering Rheology", Oxford University Press, Oxford, 1992.

[8] C.V. Vradis and J.K. Hammad, Strongly Coupled Block-Implicit Solution Technique for Non-Newtonian Convective Heat Transfer Problems, Numerical Heat Transfer, 33 (1998), 79-97. 